\title{
Using Multigraphs to Study the Interaction between Opposition, Implication and Duality Relations in Logical Squares
}

\author{
Lorenz Demey ${ }^{1[0000-0002-0176-1958]}$ and Hans Smessaert ${ }^{20000-0002-8186-0170]}$ \\ ${ }^{1}$ Center for Logic and Philosophy of Science, KU Leuven \\ lorenz. demey@kuleuven. be \\ 2 Department of Linguistics, KU Leuven \\ hans.smessaert@kuleuven.be
}

\begin{abstract}
This paper uses multigraphs to study the interaction between opposition, implication and duality relations in classical and degenerate logical squares. We show, first of all, that opposition and implication are highly symmetrical in their interaction with each other. Secondly, opposition and implication also display a higher-order symmetry, in the sense that they fulfill highly similar roles in their respective interactions with duality. Thirdly, we show that all these symmetries hold for classical squares as well as for degenerate squares.
\end{abstract}

Keywords: square of opposition · logical square $\cdot$ duality · opposition · implication · multigraph · logical geometry.

\section{Introduction}

Logical geometry studies Aristotelian diagrams, such as the square of opposition and its various extensions and generalizations. These diagrams represent a number of formulas from some logical system, and the various relations holding between them. In this context, diagrammatic reasoning consists in a form of 'diagram chasing' 1]; for example, in a well-known reasoning task, one is presented with a square of opposition and the truth value of one of its formulas, and is then asked to determine the truth values of the three other formulas in the square, by making use of the Aristotelian relations that are present in the square [9]13.

A well-known phenomenon for this type of diagrams is that multiple (types of) relations may simultaneously obtain among the formulas of a given diagram. For example, consider the well-known modal square shown in Fig 1(a) [10. As far as the Aristotelian relations are concerned, the formulas $\square p$ and $\square \neg p$ in this diagram are each other's contraries: they cannot be true together, but they can be false together. However, as far as the duality relations are concerned, these same two formulas are each other's internal negation: they result from applying the modal $\square$-operator to resp. $p$ and its negation $\neg p$. The formulas in the square in Fig. 1.(a) thus simultaneously stand in Aristotelian and duality relations. By contrast, the formulas in the classical square in Fig. 1(b) [2] stand in Aristotelian relations to each other, but not in any duality relations. 

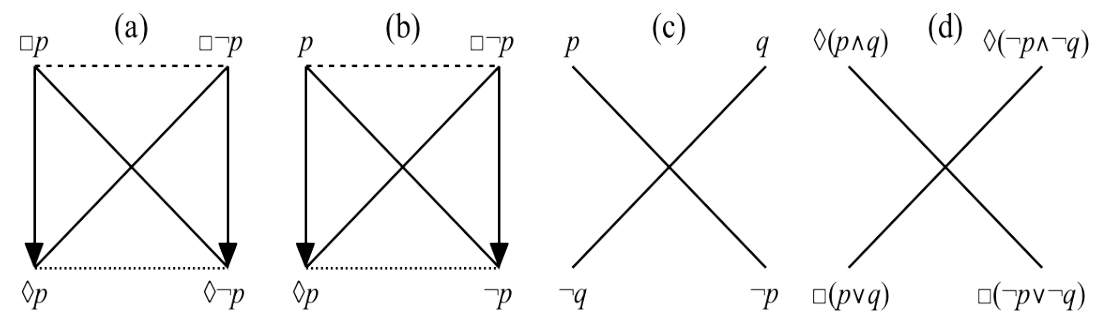

Fig. 1. (a-b) Two classical squares from resp. [10] and [2] (c-d) two degenerate squares from resp. 4 and 11 .

The two diagrams in Fig. 1 (a-b) are examples of classical squares. It is wellknown in logical geometry that next to the classical squares, there is exactly one other type of logical square, viz. the degenerate squares [4. Degenerate squares often occur as subdiagrams inside a larger diagram, e.g. a Buridan octagon [11. Apart from the contradictory relations on their diagonals, degenerate squares do not exhibit any Aristotelian relations. With regard to duality, there is again some variation. On the one hand, the degenerate square in Fig. 11(c) does not exhibit any duality relations, apart from the external negations on its diagonals. By contrast, the degenerate square in Fig. 1(d) does exhibit the full range of duality relations; for example, $\diamond(p \wedge q)$ and $\diamond(\neg p \wedge \neg q)$ are each other's internal negation, because these two formulas result from applying the binary operator $\diamond(\cdot \wedge \cdot)$ to resp. $p, q$ and their negations $\neg p, \neg q 1^{1}$

Coherent sets of logical relations are sometimes called geometries [15]. For example, the Aristotelian relations of contradiction, contrariety, subcontrariety and subalternation constitute the Aristotelian geometry $(\mathcal{A G})$. Similarly, one can also define the duality geometry $(\mathcal{D G})$, the opposition geometry $(\mathcal{O G})$ and the implication geometry $(\mathcal{I} \mathcal{G})$; precise definitions will be offered later in the paper.

The interaction between these different geometries turns out to be quite subtle, which is illustrated by the fact that a single diagram (e.g. Fig. 1(a)) can simultaneously represent multiple geometries. Consequently, these geometries and their interactions have been studied extensively in recent years [3/5/6/7/14 15]16. In particular, in 8 we proposed multigraphs as a new tool to investigate the interaction between $\mathcal{A G}$ and $\mathcal{D G}$ in several logical squares and octagons.

The overarching goal of the present paper is to show that multigraphs are by no means limited to $\mathcal{A G}$ and $\mathcal{D G}$, but can also be used to fruitfully study the interactions between other geometries. In particular, we will investigate the interactions (i) between $\mathcal{O G}$ and $\mathcal{I G}$, (ii) between $\mathcal{O G}$ and $\mathcal{D G}$, and (iii) between $\mathcal{I G}$ and $\mathcal{D G}$, in classical and degenerate squares ${ }^{2}$ We will even construct multigraphs that exhibit the interactions between these three geometries $(\mathcal{O G}, \mathcal{I G}$ and

\footnotetext{
${ }^{1}$ Note that $\diamond(\cdot \wedge \cdot)$ can be seen as the composition of the modal $\diamond$-operator and the Boolean $\wedge$-operator, and thus also gives rise to more complex types of duality behavior 3678 . However, in this paper we will simply view $\diamond(\cdot \wedge \cdot)$ as a single operator, which exhibits the simplest type of duality behavior (cf. $\mathcal{D} \mathcal{G}$ in Section 2 ).

${ }^{2}$ We thus use one type of diagrams (multigraphs) to study another (logical squares)!
} 
$\mathcal{D G})$ all at once. These investigations yield three main results. First of all, $\mathcal{O G}$ and $\mathcal{I G}$ are highly symmetrical in their interaction with each other. Secondly, $\mathcal{O G}$ and $\mathcal{I G}$ also display a higher-order symmetry, in the sense that these two geometries fulfill highly similar roles in their respective interactions with $\mathcal{D G}$. Thirdly, all these symmetries hold for classical as well as degenerate squares.

The paper is organized as follows. Section 2 surveys the required theoretical background. Section 3 uses multigraphs to study the interaction between $\mathcal{O G}, \mathcal{I} \mathcal{G}$ and $\mathcal{D G}$ in classical squares, and Section 4 does the same for degenerate squares. Section 5 wraps things up, and mentions some questions for further research.

\section{Theoretical Background}

We start by introducing the various logical geometries that will be studied in this paper. Logical relations can be characterized with various degrees of abstractness and generality [577. For our current purposes, it will suffice to stick to the traditional, informal definitions. Two statements $\varphi$ and $\psi$ are said to be

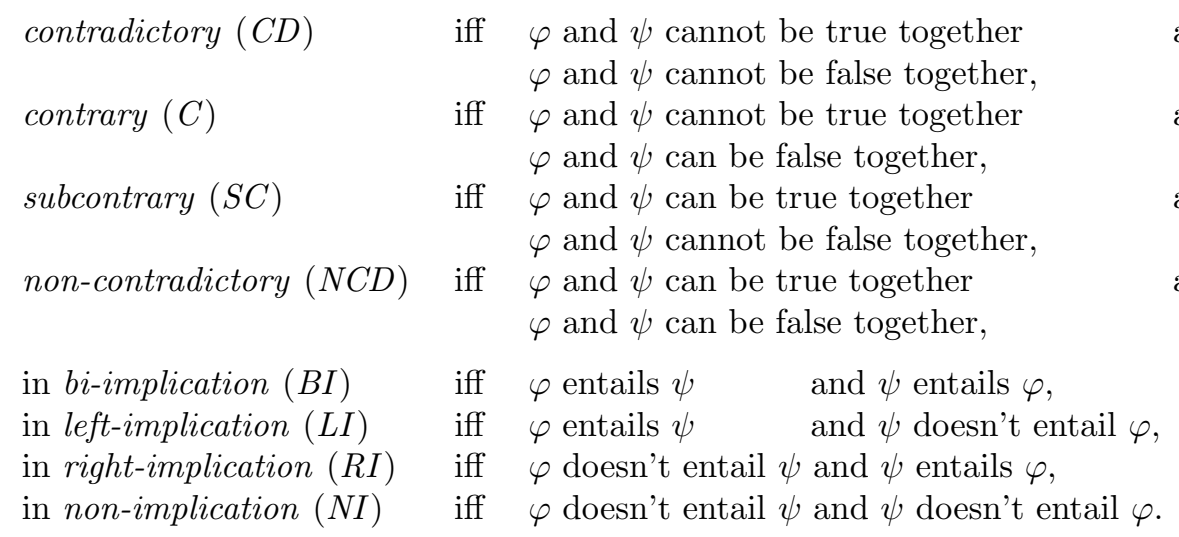

The first four of these relations constitute the opposition geometry, i.e. $\mathcal{O G}:=$ $\{C D, C, S C, N C D\}$. The next set of four relations is the implication geometry, i.e. $\mathcal{I G}:=\{B I, L I, R I, N I\}$. Both of these geometries can naturally be ordered in terms of their information levels [15]: $N C D$ and $N I$ are the least informative relations of $\mathcal{O G}$ and $\mathcal{I G}$, respectively, $C D$ and $B I$ are their most informative relations, and $C, S C, L I$ and $R I$ occupy intermediate positions. Finally, we define the Aristotelian geometry $\mathcal{A G}:=\{C D, C, S C, L I\} \subseteq \mathcal{O G} \cup \mathcal{I G}$, and note that it is 'hybrid' between $\mathcal{O G}$ and $\mathcal{I G}$ in an information-optimizing fashion [15].

We now turn to the duality relations. Suppose that $\varphi$ and $\psi$ are the results of applying $n$-ary operators $O_{\varphi}$ and $O_{\psi}$ to $n$ propositions $\alpha_{1}, \ldots, \alpha_{n}$, i.e. $\varphi \equiv$ $O_{\varphi}\left(\alpha_{1}, \ldots, \alpha_{n}\right)$ and $\psi \equiv O_{\psi}\left(\alpha_{1}, \ldots, \alpha_{n}\right)$. We say that $\varphi$ and $\psi$ are each other's

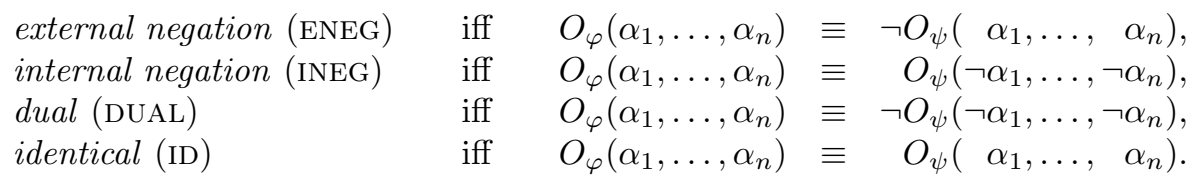



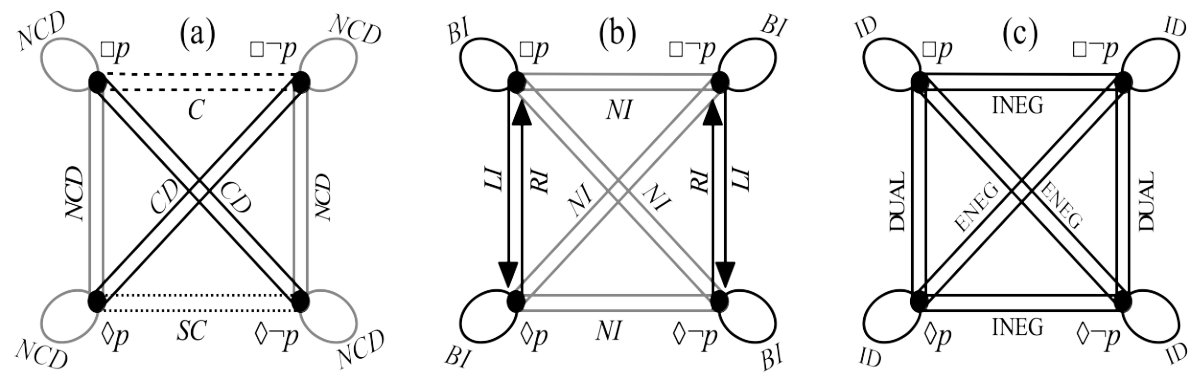

Fig. 2. (a) Opposition, (b) implication and (c) duality relations in the classical square shown in Fig. 1 (a).

These relations constitute the duality geometry, i.e. $\mathcal{D} \mathcal{G}:=\{$ ID, ENEG, INEG, DUAL $\}$, which has also been studied extensively in recent years [36[14]16].

In order to study the interaction between $\mathcal{A G}$ and $\mathcal{D G}$, we previously introduced the notion of an $\mathcal{A G} / \mathcal{D G}$-multigraph 8 . This notion can easily be generalized as follows. Let $D$ be a diagram that simultaneously represents geometries $\mathcal{G}, \mathcal{G}^{\prime} \in\{\mathcal{A G}, \mathcal{O G}, \mathcal{I} \mathcal{G}, \mathcal{D G}\}$. The $\mathcal{G} / \mathcal{G}^{\prime}$-multigraph for $D$ is defined to be a multigraph [12] with the elements of $\mathcal{G} \cup \mathcal{G}^{\prime}$ as its vertices, and precisely as many edges between vertices $R \in \mathcal{G}$ and $R^{\prime} \in \mathcal{G}^{\prime}$ as there are pairs of formulas in $D$ that simultaneously stand in the relations $R$ and $R^{\prime}$. Informally, the $\mathcal{G} / \mathcal{G}^{\prime}$-multigraph for $D$ represents how often each combination of a $\mathcal{G}$ - and a $\mathcal{G}^{\prime}$-relation occurs in $D$, and can thus help to shed light on the interaction between $\mathcal{G}$ and $\mathcal{G}^{\prime}$.

Since multigraphs are crucially concerned with numbers of (combinations of) logical relations in a given diagram, it is important to be explicit about how these relations will be counted. For example, a logical square has 4 formulas, and is therefore usually said to have $\left(\begin{array}{l}4 \\ 2\end{array}\right)=\frac{4 \times(4-1)}{2}=6$ relations (in visual terms: the 4 sides of the square and its 2 diagonals). In this calculation, the division by 2 captures the idea that the order of the formulas does not matter. However, looking at the definition of $\mathcal{I} \mathcal{G}$, it should be clear that order matters after all: $L I(\varphi, \psi)$ iff $R I(\psi, \varphi)$. Furthermore, the subtraction of 1 captures the idea that we are excluding identical pairs $(\varphi, \varphi)$. However, looking at the definitions of $\mathcal{O G}$, $\mathcal{I G}$ and $\mathcal{D G}$, it should be clear that such pairs should be kept on board after all: $N C D(\varphi, \varphi), B I(\varphi, \varphi)$ and $\operatorname{ID}(\varphi, \varphi)$. Consequently, in the remainder of this paper, we will count the number of relations in a logical square as $4 \times 4=16$.

\section{Classical Logical Squares}

The classical modal square for $\{\square p, \square \neg p, \diamond p, \diamond \neg p\}$, which was already shown in Fig. 1(a), simultaneously exhibits $\mathcal{O G}, \mathcal{I} \mathcal{G}$ and $\mathcal{D G}$. Consider, for example, the formulas $\square p$ and $\square \neg p$ : we simultaneously have $C(\square p, \square \neg p), N I(\square p, \square \neg p)$ and INEG $(\square p, \square \neg p)$. All $\mathcal{O} \mathcal{G}$-, $\mathcal{I} \mathcal{G}$ - and $\mathcal{D G}$-relations among $\square p, \square \neg p, \diamond p$ and $\diamond \neg p$ are shown in Fig. 2(a), (b) and (c), respectively. 
(a)

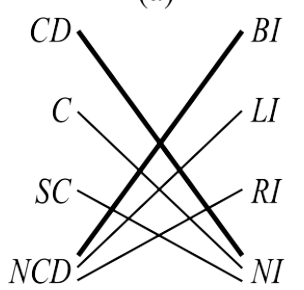

(b)

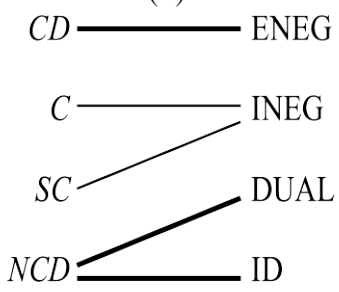

(c)

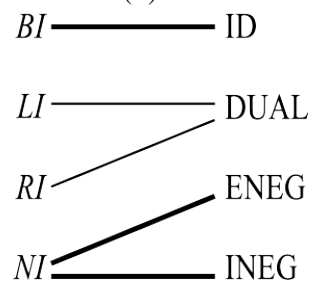

Fig. 3. (a) $\mathcal{O G} / \mathcal{I} \mathcal{G}$-, (b) $\mathcal{O G} / \mathcal{D G}$ - and (c) $\mathcal{I} \mathcal{G} / \mathcal{D G}$-multigraphs for the classical square shown in Fig. 1 (a). Thin and thick edges represent combinations of relations that cooccur resp. 2 and 4 times.

Counting the various combinations of opposition and implication relations in this square, we obtain the $\mathcal{O G} / \mathcal{I} \mathcal{G}$-multigraph shown in Fig. 3(a). For example, this multigraph indicates that there are $4 C D / N I$-combinations, viz. $(\square p, \diamond \neg p)$, $(\diamond \neg p, \square p),(\diamond p, \square \neg p)$ and $(\square \neg p, \diamond p)$. Note that this multigraph exhibits perfect vertical symmetry, which means that $\mathcal{O G}$ and $\mathcal{I} \mathcal{G}$ play very similar roles within the modal square. This vertical symmetry can be characterized in purely informational terms: the least informative relation from one geometry co-occurs precisely with the three informative relations from the other geometry. More concretely, $N C D \in \mathcal{O G}$ co-occurs with $B I, L I, R I \in \mathcal{I G}$, and vice versa, $N I \in \mathcal{I} \mathcal{G}$ co-occurs with $C D, C, S C \in \mathcal{O G}$. This symmetry between $\mathcal{O G}$ and $\mathcal{I} \mathcal{G}$ is explained in more detail in Theorems 2, 5 and 6 of [15].

We now turn to the interaction between $\mathcal{O G}$ and $\mathcal{D G}$ in the modal square, which is summarized by the $\mathcal{O G} / \mathcal{D G}$-multigraph shown in Fig. 3. (b). For example, this multigraph indicates that there are $2 C$ /NEG-combinations, viz. $(\square p, \square \neg p)$ and $(\square \neg p, \square p$ ). While there is a clear correspondence between $C D$ and ENEG, we see that INEG co-occurs with $C$ as well as $S C$, and $N C D$ co-occurs with DUAL as well as ID. This shows that the interaction between $\mathcal{O G}$ and $\mathcal{D G}$ is more subtle than is sometimes thought, especially by authors who come close to outright identifying opposition and duality relations $[17$.

In a similar fashion, we can also construct the $\mathcal{I G} / \mathcal{D G}$-multigraph, which is shown in Fig. 3(c). For example, this multigraph indicates that there are 2 $L I /$ DUAL-combinations, viz. $(\square p, \diamond p)$ and $(\square \neg p, \diamond \neg p)$. While there is a clear correspondence between $B I$ and ID, we see that DUAL co-occurs with $L I$ as well as $R I$, and $N I$ co-occurs with ENEG as well as INEG. Most importantly, note that the $\mathcal{I G} / \mathcal{D G}$-multigraph in Fig. 3(c) is isomorphic to the $\mathcal{O G} / \mathcal{D G}$-multigraph in Fig. 3(b), through the isomorphism $f: \mathcal{O G} \cup \mathcal{D G} \rightarrow \mathcal{I} \mathcal{G} \cup \mathcal{D G}$ :

$$
\begin{array}{r|cccccccc}
R \in \mathcal{O G} \cup \mathcal{D G} & C D & C & S C & N C D & \text { ENEG } & \text { INEG } & \text { DUAL } & \text { ID } \\
\hline f(R) \in \mathcal{I} \mathcal{G} \cup \mathcal{D G} & B I & L I & R I & N I & \text { ID } & \text { DUAL } & \text { ENEG } & \text { INEG }
\end{array}
$$

This isomorphism is logically meaningful, in that it corresponds to negating a relation's second argument: for all $R \in \mathcal{O G} \cup \mathcal{D G}$ it holds that $R(\varphi, \psi)$ iff $f(R)(\varphi, \neg \psi)$; cf. Lemma 3 of [15]. For example, we have $C D(\varphi, \psi)$ iff $B I(\varphi, \neg \psi)$, and also $\operatorname{EnEG}(\varphi, \psi)$ iff $\operatorname{ID}(\varphi, \neg \psi)$. Now consider the $4 C D /$ ENEG-combinations 

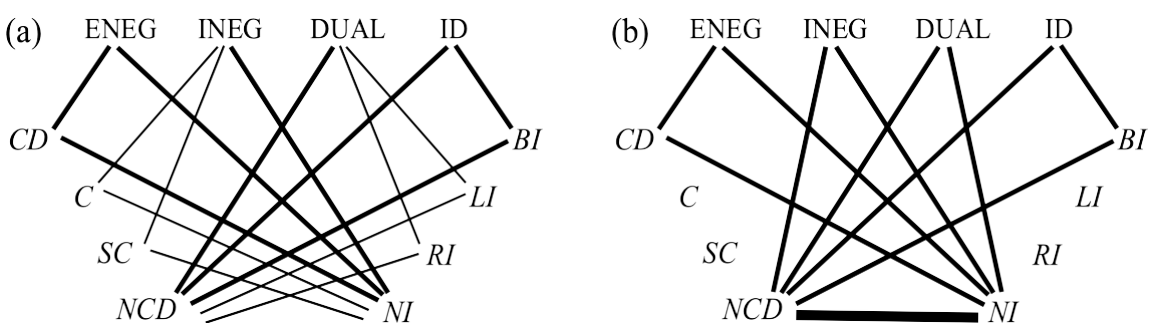

Fig. 4. $\mathcal{O G} / \mathcal{I} \mathcal{G} / \mathcal{D G}$-multigraphs for (a) the classical square shown in Fig. 11(a) and (b) the degenerate square shown in Fig. 1(d). Thin, thick and very thick edges represent combinations of relations that co-occur resp. 2, 4 and 8 times.

from the $\mathcal{O G} / \mathcal{D} \mathcal{G}$-multigraph in Fig. 3 (b), viz., $(\square p, \diamond \neg p),(\diamond \neg p, \square p),(\diamond p, \square \neg p)$ and $(\square \neg p, \diamond p)$. Systematically negating the second argument yields $(\square p, \neg \diamond \neg p)$, $(\diamond \neg p, \neg \square p),(\diamond p, \neg \square \neg p)$ and $(\square \neg p, \neg \diamond p)$, which are precisely the $4 B I /$ ID-combinations from the $\mathcal{I} \mathcal{G} / \mathcal{D G}$-multigraph in Fig. 3 (c).

We thus find that $\mathcal{O G}$ and $\mathcal{I G}$ are not only symmetrical in their interaction with each other (cf. Fig. 3(a)), but also in their respective interactions with $\mathcal{D G}$ (cf. the isomorphism between Figs. 3(b) and (c)). To summarize these findings, we combine the three separate multigraphs from Fig. 3 into one large $\mathcal{O G} / \mathcal{I} \mathcal{G} / \mathcal{D G}$-multigraph, which is shown in Fig. 4(a). This multigraph clearly reveals the highly symmetrical nature of the mutual interactions between $\mathcal{O G}$, $\mathcal{I G}$ and $\mathcal{D G}$ in the classical logical square in Fig. 1 (a). The underlying reasons and implications of this symmetry will need to be investigated in far more detail.

We now briefly consider the modal square for $\{p, \neg p, \diamond p, \square \neg p\}$, which was shown in Fig. 1(b). This alternative square simultaneously exhibits $\mathcal{O G}$ and $\mathcal{I} \mathcal{G}$, but not $\mathcal{D G}$. For example, we simultaneously have $N C D(p, \diamond p)$ and $L I(p, \diamond p)$, but $p$ and $\diamond p$ do not stand in any duality relation. The $\mathcal{O G} / \mathcal{I} \mathcal{G}$-multigraph for this alternative square is isomorphic to the one in Fig. 3(a), since both are $\mathcal{O G} / \mathcal{I} \mathcal{G}$ multigraphs for classical squares. Finally, since this alternative square does not exhibit any duality relations except for ENEG on its diagonals, it does not make much sense to construct $\mathcal{O G} / \mathcal{D G}$ - or $\mathcal{I} \mathcal{G} / \mathcal{D G}$-multigraphs for it.

\section{Degenerate Logical Squares}

We now turn to the degenerate logical squares, starting with the one shown in Fig. 1.(c). This degenerate square simultaneously exhibits $\mathcal{O G}$ and $\mathcal{I G}$, but not $\mathcal{D G}$. Consider, for example, the formulas $p$ and $q$ : we simultaneously have $N C D(p, q)$ and $N I(p, q)$, but these two formulas do not stand in any duality relation. All $\mathcal{O G}$ - and $\mathcal{I} \mathcal{G}$-relations in this square are shown in Fig. 5 (a) and (b), respectively, while its $\mathcal{O G} / \mathcal{I} \mathcal{G}$-multigraph in shown in Fig. 5(c). Note that this multigraph again exhibits perfect vertical symmetry. In particular, the least informative relations of $\mathcal{O G}$ and $\mathcal{I G}$ (viz. $N C D$ and $N I$ ) co-occur with each other, which explains the absence of any Aristotelian relations on the four sides of the degenerate square (cf. Theorems 7 and 8 of [15]). 

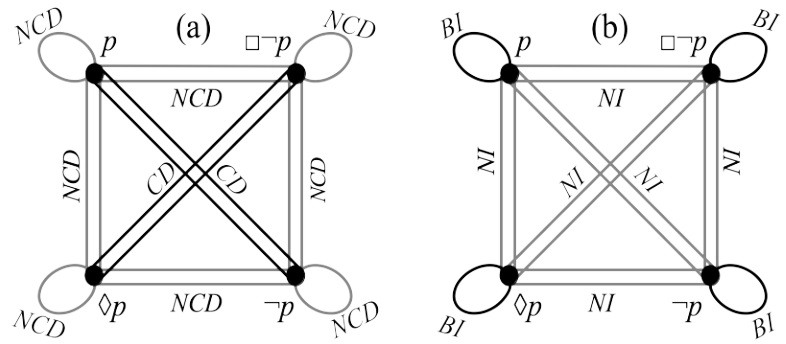

(c)

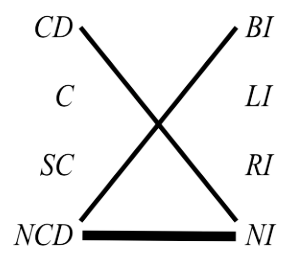

Fig. 5. (a) Opposition relations and (b) implication relations in the degenerate square shown in Fig. 1(c); (c) $\mathcal{O G} / \mathcal{I} \mathcal{G}$-multigraph for this degenerate square. Thick and very thick edges represent combinations of relations that co-occur resp. 4 and 8 times.

(a)

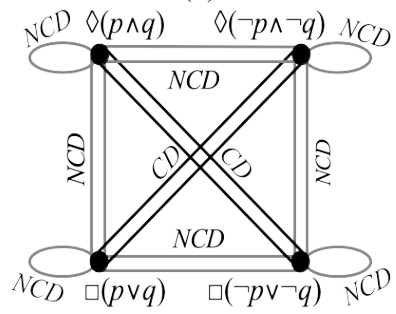

(b)

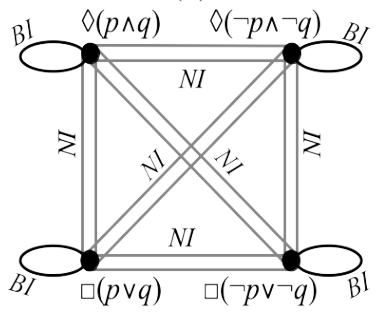

(c)

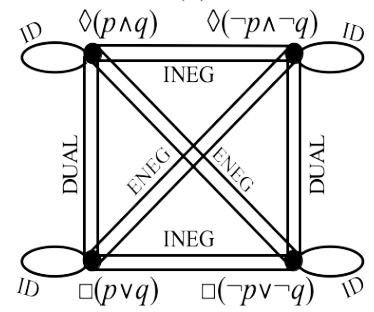

Fig. 6. (a) Opposition, (b) implication and (c) duality relations in the degenerate square shown in Fig. 1(d).

Since the degenerate square in Fig. 1(c) does not exhibit any duality relations except for ENEG on its diagonals, it does not make much sense to construct $\mathcal{O G} / \mathcal{D G}$ - or $\mathcal{I} \mathcal{G} / \mathcal{D G}$-multigraphs for it. By contrast, the degenerate square in Fig. 1(d) not only exhibits opposition and implication, but also duality relations, as shown in Fig. 6. Consequently, we can now construct not only an $\mathcal{O G} / \mathcal{I} \mathcal{G}$-, but also $\mathcal{O G} / \mathcal{D G}$ - and $\mathcal{I} \mathcal{G} / \mathcal{D G}$-multigraphs. In particular, the $\mathcal{O G} / \mathcal{I} \mathcal{G}$-multigraph is isomorphic to the one shown in Fig. 5 (c), since both are $\mathcal{O G} / \mathcal{I} \mathcal{G}$-multigraphs for degenerate logical squares. Furthermore, the $\mathcal{O G} / \mathcal{D G}$ - and $\mathcal{I} \mathcal{G} / \mathcal{D G}$-multigraphs again turn out to be isomorphic to each other. For reasons of space, however, we only show the 'combined' $\mathcal{O G} / \mathcal{I} \mathcal{G} / \mathcal{D G}$-multigraph; cf. Fig. 4(b). Once again, this multigraph clearly reveals the highly symmetrical nature of the mutual interactions between $\mathcal{O G}, \mathcal{I G}$ and $\mathcal{D G}$ in the degenerate square in Fig. $1(\mathrm{~d})$.

\section{Conclusion}

In this paper we have used multigraphs to investigate the interactions between $\mathcal{O G}, \mathcal{I} \mathcal{G}$ and $\mathcal{D G}$ in classical as well as degenerate logical squares. First of all, we have shown that $\mathcal{O G}$ and $\mathcal{I} \mathcal{G}$ are highly symmetrical in their interaction with each other — cf. Fig. 3(a). Furthermore, $\mathcal{O G}$ and $\mathcal{I G}$ also display a higherorder symmetry, in the sense that these two geometries fulfill highly similar 
roles in their respective interactions with $\mathcal{D G}$ - cf. the isomorphic multigraphs in Figs. 3(b-c). Finally, these symmetries not only hold for classical squares, but also for degenerate squares - compare Figs. 3(a) and 4 (a) with resp. Figs. 5(c) and $4(\mathrm{~b})$. These results clearly illustrate the potential fruitfulness of multigraphs for studying the interactions between various logical geometries.

In future work, we will extend this research line along three complementary dimensions. First of all, we will explore alternative multigraph visualizations, which optimize different diagrammatic criteria (e.g. central symmetry, fewer edge crossings, etc.). Secondly, we will study interactions involving the Aristotelian geometry $(\mathcal{A G})$, in particular the interactions $\mathcal{A G} / \mathcal{O G}$ and $\mathcal{A G} / \mathcal{I G}$. Thirdly, we will also look at diagrams larger than squares, e.g. logical hexagons and octagons.

Acknowledgements We would like to thank Margaux Smets for her valuable feedback on an earlier version of this paper. The research of the first author was supported by a Postdoctoral Fellowship of the Research Foundation-Flanders (FWO) and a Research Professorship (BOFZAP) from KU Leuven.

\section{References}

1. De Toffoli, S.: 'Chasing' the diagram - the use of visualizations in algebraic reasoning. Review of Symbolic Logic 10, 158-186 (2017)

2. Dedelley, J.: Summulae Logicae (Editio Tertia). Joannes Paulus Schleig (1738)

3. Demey, L.: Algebraic aspects of duality diagrams. In: Cox, P.T., Plimmer, B., Rodgers, P. (eds.) Diagrams 2012, pp. 300-302. LNCS 7352, Springer (2012)

4. Demey, L.: Computing the maximal Boolean complexity of families of Aristotelian diagrams. Journal of Logic and Computation 28, 1323-1339 (2018)

5. Demey, L.: Metalogic, metalanguage and logical geometry. Logique et Analyse 248, 453-478 (2019)

6. Demey, L., Smessaert, H.: Duality in logic and language. In: Fieser, J., Dowden, B. (eds.) Internet Encyclopedia of Philosophy. University of Tennessee (2016)

7. Demey, L., Smessaert, H.: Metalogical decorations of logical diagrams. Logica Universalis 10, 233-292 (2016)

8. Demey, L., Smessaert, H.: Aristotelian and duality relations beyond the square of opposition. In: Chapman, P., et al. (eds.) Diagrams 2018, pp. 640-656. LNCS 10871, Springer (2018)

9. Dopp, J.: Formal Logic. Joseph F. Wagner, Inc., New York City, NY (1960)

10. Fitting, M., Mendelsohn, R.L.: First-Order Modal Logic. Kluwer (1998)

11. García Cruz, J.D.: Aristotelian relations in PDL: The hypercube of dynamic oppositions. South American Journal of Logic 3, 389-414 (2017)

12. Harary, F.: Graph Theory. CRC Press (1994)

13. Hurley, P.J.: A Concise Introduction to Logic (11th ed.). Wadsworth (2012)

14. Smessaert, H.: The classical Aristotelian hexagon versus the modern duality hexagon. Logica Universalis 6, 171-199 (2012)

15. Smessaert, H., Demey, L.: Logical geometries and information in the square of opposition. Journal of Logic, Language and Information 23, 527-565 (2014)

16. Smessaert, H., Demey, L.: Duality patterns in 2-PCD fragments. South American Journal of Logic 3, 225-272 (2017)

17. Yao, Y.: Duality in rough set theory based on the square of opposition. Fundamenta Informaticae 127, 49-64 (2013) 